\title{
A stable implementation of multiple Higdon's absorbing boundary condition for Maxwell's equations
}

\author{
Rengang Shi, Xinmin Ge, Zhoutuo Wei and Shaogui Deng
}

\begin{abstract}
Stability is a crucial problem for implementation of Higdon's absorbing boundary condition (ABC). Unstable phenomena were found in the traditional implementations of Higdon's ABC, especially the 2nd-order Higdon's ABC. This letter presents a stable implementation of multiple Higdon's ABC to truncate the unbounded domain for simulating Maxwell's equations by the finite-difference time-domain method. This implementation applies Higdon's ABC to the physical domain at several layers. The multiple Higdon's ABC (MH ABC) adopts a combination of Maxwell's equations and one-way wave equation with weights. This letter presents a detailed introduction of $\mathrm{MH}$ ABC with the 2nd-order Higdon's ABC. The optimal weights are determined by numerical tests. And the MH ABC can be stably implemented with wide-band frequency. Numerical examples show that the MH ABC obtains excellent absorption effect with ultra-thin ABC layers, such as three. 3D comparisons between the MH ABC and Betz-Mittra $\mathrm{ABC}$ are also presented.
\end{abstract}

Index Terms-Finite-difference time-domain, Stability, Multiple, Higdon's absorbing boundary condition, Maxwell's equations.

\section{INTRODUCTION}

W Hen using finite-difference time-domain (FDTD) method to simulate electromagnetic fields in unbounded domain, an absorbing boundary condition (ABC) should be used to truncate the space. Higdon's ABC [1], [2] is one of the effective and promising absorbing boundary conditions. Based on one-way wave Eqn.(1), Higdon presented an annihilation operator with backward difference scheme on the truncation boundary.

$$
\left[\prod_{j=1}^{p}\left(\cos \left(\alpha_{j}\right) \frac{\partial}{\partial t}-c \frac{\partial}{\partial x}\right)\right] u=0
$$

It is easy to extend the Higdon's ABC to higher orders by higher operator $p$, where choosing special angles can increase the angular spectrum with reflection error in low level. By a reformulation of Higdon's ABC, Givoli and Neta [3] presented a convenient implement of high-order Higdon's $\mathrm{ABC}$ where discretization only for first-order derivatives are needed. Based on minimizing the error between approximate and exact dispersion relations, Peng et al. [4] presented a

This work was supported by the Major Scientific and Technological Projects of CNPC under Grant (ZD2019-184-001) and the Fundamental Research Funds for the Central Universities(20CX05011A).

R. Shi is with the College Of Science, China University of Petroleum, QingDao, 266580, China. (email:shirg@upc.edu.cn)

X. Ge, Z. wei and S. Deng are with the School of Geosciences, China University of Petroleum (East China), Qingdao, 266580, China. fourth-order Higdon's ABC with optimized coefficients for the simulation of acoustic equation, where numerical test show its best absorbing effect and efficiency among several artificial absorbing boundaries. Based on physical background, Berenger et al. [5] found that the first-order Higdon's ABC can be explained by a traveling wave absorbed in a dispersive medium.

Although the Higdon's ABC [6] was proved to be energystable up to any order on the continuous level, instability of implementing the high-order Higdon's ABC with Eqn. (1) was found in [2], [7], [8]. To make the implementation of highorder Higdon's ABC more robust, a attenuation factor $\varepsilon_{j}$ was introduced to Eqn.(1), which leads to

$$
\left[\prod_{j=1}^{p}\left(\cos \left(\alpha_{j}\right) \frac{\partial}{\partial t}-c \frac{\partial}{\partial x}+\varepsilon_{j}\right)\right] u=0 .
$$

Higdon [2] gave a thorough stability analysis for Higdon's $\mathrm{ABC}$ with the attenuation factor $\varepsilon_{j}$, and shown its effect with certain attenuation factor. Betz and Mittra [9] presented a stable implementation of 2nd-order Higdon's ABC with one attenuation factor, which works on both traveling and evanescent waves.

This letter presents a multiple Higdon's ABC (MH ABC) to stably implement Higdon's ABC without attenuation factor. The one-way wave equation adopted by $\mathrm{MH} \mathrm{ABC}$ in this letter is

$$
\left[\prod_{j=1}^{p}\left(\cos \left(\alpha_{j}\right) \frac{\partial}{\partial t}+c \frac{\partial}{\partial \mathbf{n}}\right)\right] u=0
$$

where the direction $\mathbf{n}$ of spatial derivative is different from Eqn.(1). The Higdon's ABC will be applied not only on the outmost boundary but also on several layers in the physical domain where Maxwell's equations combine Eqn. (3) with certain weight at each layer. At boundaries of each layer of MH ABC, the direction of spatial derivative in Eqn.(3) is the same as that in Eqn.(1). For example, at the left boundary, spatial derivative is along the negative $x$-direction, that is,

$$
\frac{\partial}{\partial \mathbf{n}}=-\frac{\partial}{\partial x} \text {. }
$$

But at the corners of the truncated domain, the spatial derivative is along the diagonal which wasn't concerned in traditional implementation of Higdon's ABC. This letter takes 2D TEz wave for example to implement $\mathrm{MH} \mathrm{ABC}$ with 2nd-order Higdon's ABC. 


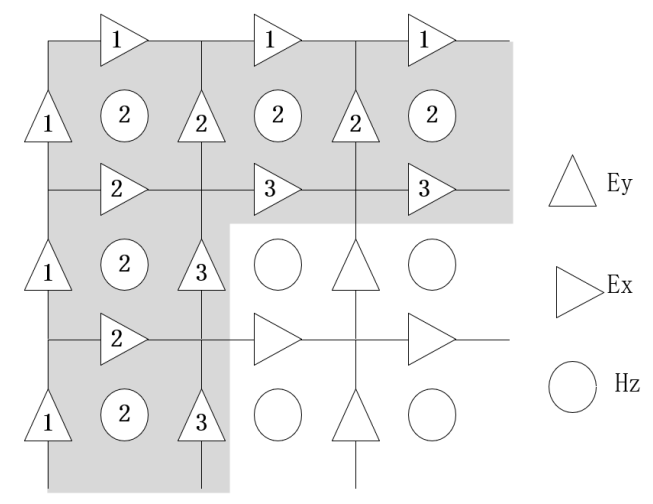

Fig. 1. The left-up corner of MH ABC layers in 2D TEz case.

\section{THE MH ABC}

This section presents the $\mathrm{MH} \mathrm{ABC}$ in detail. On the staggered grid in 2D TEz case, the structure of the $\mathrm{MH} \mathrm{ABC}$ at the left-up corner is shown by Fig.1, where the shade refer to the MH ABC. The number on node symbols for components denotes the layer indices where Higdon's ABC is applied. It can be found from Fig. 1 that all nodes on the outmost boundary belong to the first layer, and all nodes, half-cell away from the outmost boundary along $x$ - or $y$-direction, belong to the second layer. At each node of the grid, unknown field is obtained by a combination. For example, at node $\left(x_{1}, y_{i+1 / 2}\right)$, $E_{y}$ is the local unknown component which can be written by

$$
E_{y}=w E_{y}^{\mathrm{M}}+(1-w) E_{y}^{\mathrm{H}}
$$

where $E_{y}^{\mathrm{M}}$ and $E_{y}^{\mathrm{H}}$ satisfy

$$
\begin{aligned}
& \varepsilon \frac{\partial E_{y}^{\mathrm{M}}}{\partial t}=-\frac{\partial H_{z}^{\mathrm{M}}}{\partial x} \\
& {\left[\prod_{j=1}^{p}\left(\cos \left(\alpha_{j}\right) \frac{\partial}{\partial t}+c \frac{\partial}{\partial \mathbf{n}}\right)\right] E_{y}^{\mathrm{H}}=0 .}
\end{aligned}
$$

and the weight $w(0 \leq w \leq 1)$ varies along direction $\mathbf{n}=-x$. In this letter, components with superscript $\mathrm{M}$, such as $E_{y}^{\mathrm{M}}$, denote that these components satisfy Maxwell's equations, and components with superscript $\mathrm{H}$, such as $E_{y}^{\mathrm{H}}$, denote that these components satisfy one-way wave equation. At node $\left(x_{1+1 / 2}, y_{1+1 / 2}\right), H_{z}$ is the local unknown component which can be written as

$$
H_{z}=w H_{z}^{\mathrm{M}}+(1-w) H_{z}^{\mathrm{H}}
$$

where $H_{z}^{\mathrm{M}}$ and $H_{z}^{\mathrm{H}}$ satisfy

$$
\begin{aligned}
& \mu \frac{\partial H_{z}^{\mathrm{M}}}{\partial t}=\frac{\partial E_{x}^{\mathrm{M}}}{\partial y}-\frac{\partial E_{y}^{\mathrm{M}}}{\partial x} \\
& {\left[\prod_{j=1}^{p}\left(\left(\cos \left(\alpha_{j}\right) \frac{\partial}{\partial t}+c \frac{\partial}{\partial \mathbf{n}}\right)\right)\right] H_{z}^{\mathrm{H}}=0}
\end{aligned}
$$

and $\mathbf{n}$ is the outward diagonal. The weight $w$ is defined by

$$
w=r_{1}\left(\frac{(\rho-1) h / 2}{d h / 2}\right)^{m}
$$

where $0<r_{1}<1$ is a ratio, $m$ is the power, $h$ is the grid size, the distance between adjacent $\mathrm{MH} \mathrm{ABC}$ layers is $h / 2$, $d h / 2$ is total thickness of $\mathrm{MH} \mathrm{ABC}, \rho_{0}$ is the total number of nodes in the direction perpendicular to layers, and $(\rho-1) h / 2$ is the distance of one layer from outermost boundary and $1 \leq$ $\rho \leq \rho_{0}$ is integer. With $d<1$, the $\mathrm{MH} \mathrm{ABC}$ will become the traditional Higdon's $\mathrm{ABC}$. So, the MH ABC scheme will cost more memory than the traditional Higdon's scheme when $d>1$. But the extra cost is little with ultra-thin $\mathrm{MH} \mathrm{ABC}$ layers, such as $\rho_{0}=3$.

\section{SCHEME FOR MH ABC}

This section presents FDTD scheme for the MH ABC. In the whole domain, Maxwell's equations are solved by the Yee's FDTD scheme [10]. For one-way wave equation (3), the Higdon's annihilation operator is used. For any grid function $U_{i, j}^{n+1}$, we define the backward space-shift operator as

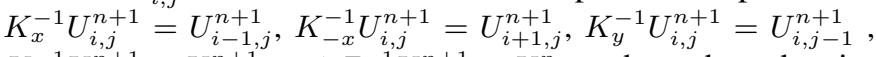
$K_{-y}^{-1} U_{i, j}^{n+1}=U_{i, j+1}^{n+1}$ and $Z^{-1} U_{i, j}^{n+1}=U_{i, j}^{n}$, where the subscript $x$ or $-x$ indicates the direction of operator. For components $E_{x}, E_{y}$ and $H_{z}$, the Higdon's annihilation operator can be directly used when the direction of one-way equation (3) is $x$, $-x, y$ or $-y$. For example, the 2nd-order Higdon's annihilation operator for Eqn. (7) can be written as

$$
\prod_{j=1}^{2}\left(I+K_{-x}^{-1} Z^{-1}+\gamma_{j} K_{-x}^{-1}-\gamma_{j} Z^{-1}\right) E_{y}^{\mathrm{H}}=0
$$

where

$$
\gamma_{j}=\frac{c \Delta t-h \cos \alpha_{j}}{c \Delta t+h \cos \alpha_{j}}
$$

For Eqn. (10), the outward normal direction is the diagonal. In this letter, when one-way equation (3) is on the diagonal, it is approximated by summation of adjacent directions. For example, the 2nd-order Higdon's annihilation operator for Eqn. (10) is

$$
\begin{aligned}
& 0.5 \prod_{j=1}^{2}\left(I+K_{y}^{-1} Z^{-1}+\gamma_{j} K_{y}^{-1}-\gamma_{j} Z^{-1}\right) H_{z}^{\mathrm{H}}+ \\
& 0.5 \prod_{j=1}^{2}\left(I+K_{-x}^{-1} Z^{-1}+\gamma_{j} K_{-x}^{-1}-\gamma_{j} Z^{-1}\right) H_{z}^{\mathrm{H}}=0
\end{aligned}
$$

Based on the Higdon's annihilation operator, such as (12) and (13), numerical approximations for components $E_{x}, E_{y}$ and $H_{z}$ can be directly obtained.

After the approximations $\mathbf{E}^{k}=\left(E_{x}^{k}, E_{y}^{k}\right)$ and $H_{z}{ }^{k}$ at time level $k$ are obtained, $\mathbf{E}^{k+1}$ and $H_{z}{ }^{k+1}$ can be calculated by the following scheme:

a) Let $\mathbf{E}^{\mathrm{M}^{k}}=\mathbf{E}^{k}$ and $H_{z}{ }^{\mathrm{M}^{k}}=H_{z}{ }^{k}$, and obtain the approximations $\mathbf{E}^{\mathrm{M}^{k+1}}$ and $H_{z} \mathrm{M}^{k+1}$ for Maxwell's equations by the Yee's FDTD method.

b) Let $\mathbf{E}^{\mathrm{H}^{k}}=\mathbf{E}^{k}$ and $H_{z} \mathrm{H}^{k}=H_{z}{ }^{k}$, and obtain the approximations $\mathbf{E}^{\mathrm{H}^{k+1}}$ and $H_{z}{ }^{\mathrm{H}^{k+1}}$ for Higdon's equation (3) by Higdon's annihilation operator.

c) The approximations $\mathbf{E}^{k+1}$ and $H_{z}{ }^{k+1}$ at time level $k+1$ are obtained by

$$
\begin{aligned}
& \mathbf{E}^{k+1}=(1-w) \mathbf{E}^{\mathrm{H}^{k+1}}+w \mathbf{E}^{\mathrm{M}^{k+1}} \\
& H_{z}{ }^{k+1}=(1-w) H_{z}{ }^{\mathrm{H}^{k+1}}+w H_{z}{ }^{\mathrm{M}^{k+1}} .
\end{aligned}
$$




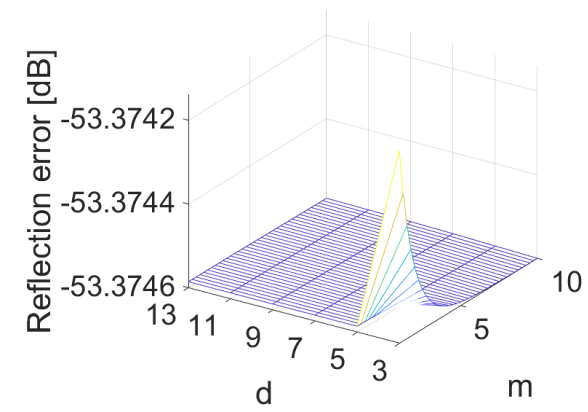

Fig. 2. Maximum reflection error over all observation points as a function of odd $d$ and $m$ with $\alpha_{1}=15 \pi / 180, \alpha_{2}=45 \pi / 180$ and $r_{1}=10^{-6}$.

\section{NUMERICAL STUDY}

To study the validity of MH ABC, 2D TEz case with Ricker source $f(t)$ in vacuum are considered in this section

$$
f(t)=\left(1-2\left(\pi f_{0}\left(t-\tau_{0}\right)\right)^{2}\right) e^{-\left(\pi f_{0}\left(t-\tau_{0}\right)\right)^{2}} .
$$

The total domain contains $220 \times 220$ cells. The source is placed at node $(110,110)$ with the magnetic-field component $H_{z}$. Take all points half-cell away from the upper inner boundary of $\mathrm{MH} A B C$ as the observation points. Reflection error in the time domain is defined as

$$
\text { Error }_{d B}=20 \log \frac{\left|H_{z}^{k}-H_{z}^{R e f \cdot}\right|}{\max _{k}\left|H_{z}^{R e f \cdot}\right|}
$$

where $H_{z}^{k}$ denotes the numerical results obtained with the

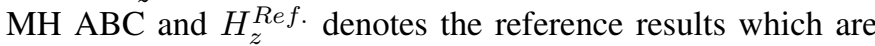
computed by a large enough domain with $2200 \times 2200$ cells .

\section{A. Parameters of $M H A B C$}

There are five parameters, $\alpha_{1}, \alpha_{2}, r_{1}, d$ and $m$, in $\mathrm{MH}$ ABC. And their impacts on reflection error will be shown by numerical tests where the error is maximum reflection error with certain varying parameters over the first 1000 time iterations among all observation points. In these tests, parameters are given by $f_{0}=35(\mathrm{~Hz}), \tau_{0}=1 / f_{0}, \alpha_{1}=15 \pi / 180$, $\alpha_{2}=45 \pi / 180, r_{1}=10^{-6}, d=3, \rho_{0}=3, m=9$, grid size $h=4.0 \times 10^{5}(\mathrm{~m})$ and time step $\Delta t=\frac{h}{\sqrt{2} c}(\mathrm{~s})$ when these parameters aren't specified.

To show the combined impact of $d$ and $m$ to reflection error, Fig. 2 shows the maximum reflection error with varying $d$ and $m$, where $d$ is integer and $\rho_{0}=d$. The reflection error with even $d$ is about double of those with odd $d$ and does not show in Fig.2. It can be found from Fig. 2 that the reflection error of $\mathrm{MH} \mathrm{ABC}$ decrease with increasing $d$ or $m$. Fig. 2 also shows that the reflection error decrease rapidly from $d=3$ to $d=5$ and slowly as $d \geq 5$. With increasing $m$, Fig. 2 illustrates that the reflection error decrease exponentially, and errors with $m>6$ are almost the same as those with $d \geq 5$. So considering the efficiency, $d=3$ and $m>6$ should be the best choice for $\mathrm{MH} \mathrm{ABC}$.

Fig.3 shows maximum reflection error with varying $r_{1}$ and $d$ where $\rho_{0}=3$ is unchanged in this test. Fig.3 illustrates that the reflection error increase rapidly as the inner boundary

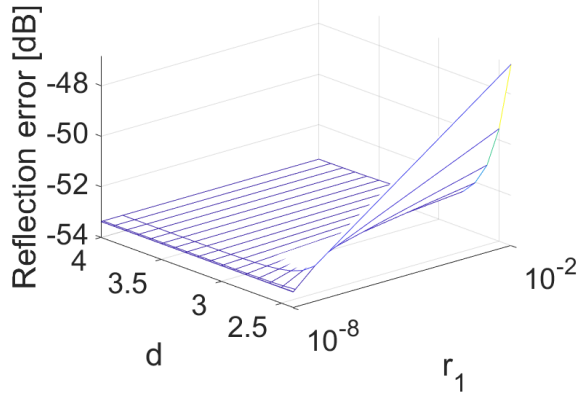

Fig. 3. Maximum relative error over all observation points as a function of $d$ and $r_{1}$ with $\alpha_{1}=15 \pi / 180, \alpha_{2}=45 \pi / 180$ and $m=9$.

move toward the second layer and decrease slowly oppositely. Fig. 3 also shows that the reflection error decrease slowly with decreasing $r_{1}$.

The impact of $\alpha_{j}$ to reflection error is shown by Fig. 4 which seems a bow with convex bottom. The minimum reflection error, about -56.12 , locates on a circle of $\alpha_{1}$ and $\alpha_{2}$,

$$
\alpha_{1}^{2}+\alpha_{2}^{2}=\frac{25 \pi^{2}}{400} .
$$

Outside this circle, the reflection error increase rapidly. And inside this circle, the reflection error increase slowly. The reflection error is -46.87 at middle of the bottom with $\alpha_{1}=0$, $\alpha_{2}=0$, which means that the absorbing effect can be improved about $20 \%$ with optimal $\alpha_{j}$.

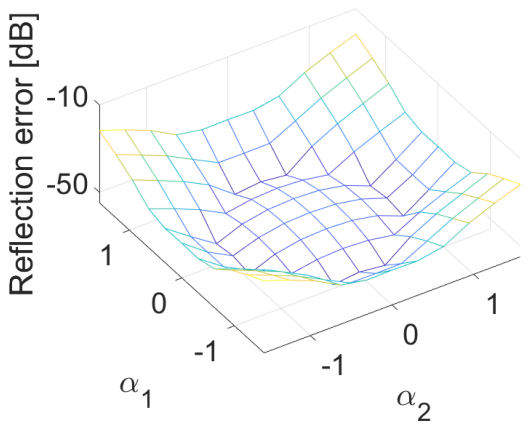

Fig. 4. Maximum relative error over all observation points as a function of $\alpha_{1}$ and $\alpha_{2}$ with $\rho_{0}=3, r_{1}=10^{-6}$ and $m=9$.

To show the impact of frequency to reflection error, we implement $\mathrm{MH}$ ABC with varying $f_{0}, h=\frac{c}{10 f_{0}}(m)$ and $\Delta t=\frac{h}{\sqrt{2} c}(s)$. The maximum reflection error over all observation points within the first 1000 time steps are shown in Fig. 5, which illustrates that the reflection error hovers around $-51.49[\mathrm{~dB}]$ with broadband frequency. In other words, the $\mathrm{MH} \mathrm{ABC}$ works well with broadband frequency. Furthermore, Eqn.(18) still works within $f_{0} \in\left[10,10^{10}\right](\mathrm{Hz})$ in our numerical tests.

\section{B. 3D Implementation}

Each slice in 3D staggered grid can be treated as a 2D structure, such as Fig.1. So the $\mathrm{MH} \mathrm{ABC}$ can be easily 


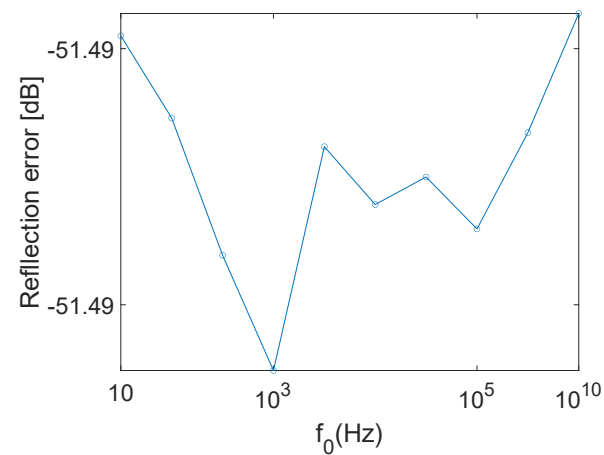

Fig. 5. Maximum relative error over all observation points as a function of frequency $f_{0}$ with $\alpha_{j}=\frac{\sqrt{2} \pi}{8}, \rho_{0}=3, r_{1}=10^{-6}$ and $m=9$.

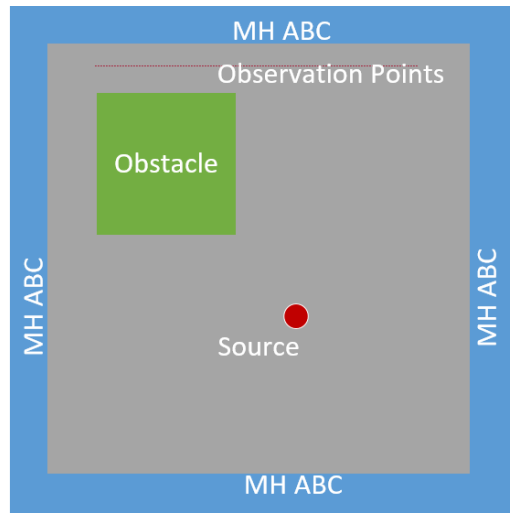

Fig. 6. FDTD space for absorption of the field scattered by an obstacle in vacuum

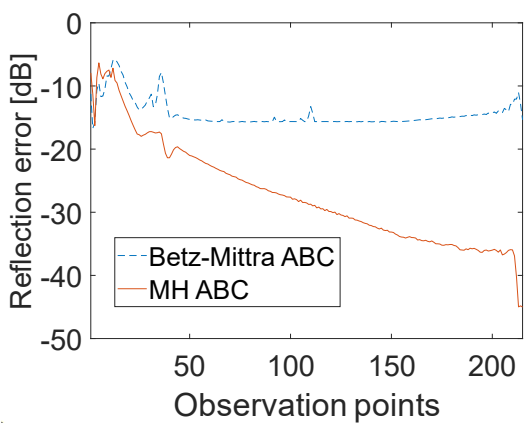

Fig. 7. Maximum reflection error of Betz-Mittra $\mathrm{ABC}$ and $\mathrm{MH} \mathrm{ABC}$ at each observation point

extended to 3D case. This section presents a 3D comparisons of $\mathrm{MH} \mathrm{ABC}$ and Betz-Mittra $\mathrm{ABC}$ [9] for absorbing signals scattered by an obstacle in vacuum.

The cuboid obstacle, with $30 \times 30 \times 200$ cells, is placed along the $z$-direction, with its long side parallel to $z$-axis, ten cells away from both boundaries in $z$-direction and two cells away from the internal face of $\mathrm{MH} \mathrm{ABC}$ in the $x$ - and $y$-direction. The obstacle is with relative dielectric constant $\varepsilon_{r}=1$ and relative magnetic permeability $\mu_{r}=1.8$. The Ricker source (16) with $f_{0}=1(\mathrm{GHz})$ is placed at the center of a plane which is perpendicular to $z$-axis and pass through point $z_{110}$. The plane is shown by Fig.6 where the observation points along $y$-direction are placed one cell away from the surface of the obstacle.

To keep the stability of Betz-Mittra $\mathrm{ABC}$, its time step is half of that used for MH ABC $\Delta t=\frac{h}{\sqrt{3} c}(s)$. Other parameters of Betz-Mittra ABC can be found in [9]. Other parameters of $\mathrm{MH} \mathrm{ABC}$ can be found in section IV-A.

The maximum reflection errors of the Betz-Mittra $\mathrm{ABC}$ and $\mathrm{MH} \mathrm{ABC}$ over the first 1000 time iterations at each observation point are shown by Fig.7, where Betz-Mittra ABC and $\mathrm{MH} \mathrm{ABC}$ show their absorbing capacity for traveling and evanescent waves. Fig.7 illustrates that reflection errors of Betz-Mittra $\mathrm{ABC}$ and $\mathrm{MH} \mathrm{ABC}$ are almost the same at observation points over the obstacle. Fig.7 also illustrates that reflection errors of $\mathrm{MH} \mathrm{ABC}$ decrease from left to right, meanwhile the reflection errors of the Betz-Mittra $A B C$ are almost invariant. At the rightmost observation points, the $\mathrm{MH}$ $\mathrm{ABC}$ almost reachs the best absorbing capacity of 2nd-order Higdon's ABC. Fig.7 shows that the attenuation factor $\varepsilon_{j}$ keeps the Betz-Mittra ABC stable and weakens the absorbing capacity.

\section{CONClusion}

This letter presents $\mathrm{MH} \mathrm{ABC}$ to stably implement Higdon's $\mathrm{ABC}$ without attenuation factor. The implementation method of 2nd-order Higdon's ABC can be easily extended to Higdon's $\mathrm{ABC}$ with any other order. The $\mathrm{MH} \mathrm{ABC}$ works well with three layers, which leads to its efficiency. The MH ABC applies Higdon's ABC at several layers, which keeps its excellent stability and absorbing capacity with wide-band frequency. Compared with Betz-Mittra $\mathrm{ABC}$, the $\mathrm{MH} \mathrm{ABC}$ shows its better stability, efficiency, and absorbing capacity for traveling and evanescent waves by numerical tests.

\section{REFERENCES}

[1] R.L. Higdon, "Absorbing boundary conditions for difference approximations to the multidimensional wave equation", Mathematics of computation, vol. 47, no. 176, pp. 437-459, Oct. 1986.

[2] R.L. Higdon, "Numerical absorbing boundary conditions for the wave equation", Mathematics of computation, vol.49, no. 179, pp.65-90. Jul. 1987.

[3] D.Givoli and B.Neta, "High-order non reflecting boundary scheme for time-dependent waves", Journal of computational physics, vo. 186, pp.24-46, 2003.

[4] S.Peng, L.zhaolun, Zh.xiaobo, et al., "The fourth-order absorbing boundary condition with optimized coefficients for the simulation of the acoustic equation", Journal Geophysics and engineering, vol.12, pp.996-1007, Dec. 2015.

[5] B. Abdulkareem, J.P. Berenger, and F. Costen et al.," An operator absorbing boundary condition for the absorption of Electromagnetic waves in dispersive media", IEEE Transactions on antennas and propagation, vol.66, no. 4, pp.2147-2150, Apr.2018.

[6] D.Baffet, and D.Givoli, "On the stability of the high-order Higdon Absorbing Boundary Conditions", Applied numerical mathematics, vol.61, no.6, pp.768-784, Feb. 2011.

[7] O.M. Ramahi, "Stable FDTD solutions with higher-order absorbing boundary conditions", Microwave and optical technology letters, vol. 15, no. 3, pp.132-134, Feb.1997.

[8] O.M. Ramahi, "Stability of absorbing boundary conditions", IEEE Transactions on Antennas and Propagation, vol.47, no. 4, pp.593-599, Apr. 1999.

[9] V. Betz and R. Mittra, "Comparison and evaluation of boundary conditions for the absorption of guided waves in an FDTD simulation", IEEE Microwave \& Guided Wave Letters, vol. 2, no.12, pp.499-501, Dec. 1992.

[10] K.S.Yee, "Numerical solution of initial boundary value problems involving Maxwell's equations in isotropic media", IEEE Transactions on Antennas \& Propagation, vol.14, no.5, pp.302-307, 1966. 